\title{
The Right Aortic Arch with Aberrant Left Subclavian Artery as a Cause of Dysphagia Lusoria
}

\author{
Koc ${ }^{1}$, Doganay $\mathrm{S}^{2}$, Gorkem $\mathrm{SB}^{3}$, Dogan $\mathrm{MS}^{4}$, Coskun $\mathrm{A}^{5}$
}

${ }^{1}$ Gonca Koc, MD, ${ }^{2}$ Selim Doganay, MD, Assoc. Prof. in Radiology, ${ }^{3}$ Sureyya Burcu Gorkem, $\mathrm{MD}$, Assist. Prof. in Radiology, ${ }^{4}$ Mehmet Sait Dogan, MD, ${ }^{5}$ Abdulhakim Coskun, MD, Prof. in Radiology. All from the Department of Paediatric Radiology, Faculty of Medicine, Erciyes University, 38039 Kayseri, Turkey

\section{Address for correspondence:}

Gonca Koc, E-mail; ggulkoc@gmail.com

\section{How to cite}

Koc G, Doganay S, Gorkem SB, Dogan MS, Coskun A. The Right Aortic Arch with Aberrant Left Subclavian Artery as a Cause of Dysphagia Lusoria. J Nepal Paediatr Soc 2016;36(1):9799.

doi: http://dx.doi.org/10.3126/jnps.v36i1.14584

This work is licensed under a Creative Commons Attribution 3.0 License.

\begin{abstract}
The right aortic arch with aberrant left subclavian artery is a rare vascular anomaly that usually does not cause any symptoms and is encountered in the adult age group incidentally. We report a paediatric case presented with dysphagia lusoria resulted from right aortic arch with aberrant left subclavian artery with the imaging findings.
\end{abstract}

Key words: Dysphagia lusoria; aberrant left subclavian artery; barium esophagography; computed tomography

\section{Introduction}

$T$ he right aortic arch with aberrant left subclavian artery is one of the obstructing vascular anomalies that may cause stridor or swallowing difficulties due to compression of either trachea or oesephagus. Approximately $0.1 \%$ of the population has a right-sided aortic arch, and nearly half of these are accompanied with an aberrant left subclavian artery ${ }^{1}$. Patients with the right aortic arch with aberrant left subclavian artery are usually diagnosed incidentally during the adulthood. The imaging modalities including barium esophagography, computed tomography angiography (CTA), and magnetic resonance angiography (MRA) are implemented to establish the definite diagnosis. Herein, we report the imaging findings of a rare paediatric case presented with dysphagia associated with right aortic arch with aberrant left subclavian artery.

\section{The Case}

A five year old boy was admitted to paediatric gastroenterology clinic with the complaints of difficulty in swallowing solid foods started with the initiation of supplementary food. On physical examination, the height and weight were revealed to be less than 3 standard deviations for age. The physical examination was otherwise unremarkable. The blood count, biochemistry, and urine tests were within normal limits. Patient was referred for the acquisition of barium oesophagography examination to rule out possible oesophageal strictures. On oesophagography an indentation about one vertebral body height on the lateral and posterior aspects of thoracic oesophagus, just superior to the carina level suggesting external compression was observed (Figure 1). There was no pre-stenotic dilatation of the 
oesophageal segment proximal to the indentation. Computed tomography angiography (CTA) performed to identify probable vascular anomalies that may cause indentation to oesophagus revealed right-sided aortic arch. Accompanying left aberrant subclavian artery was crossing posterior to oesophagus and going towards to left upper extremity after originating from aorta (Figure

2). Descending aorta was located on the right side of the

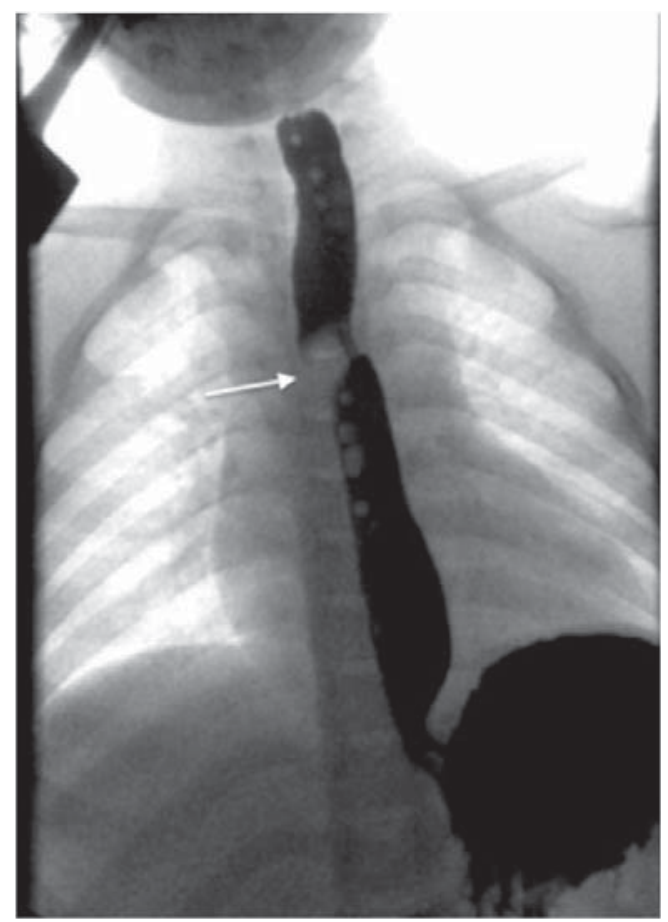

Fig 1: Barium oesophagography examination revealed indentation of thoracic esophagus on lateral (a) and posterior (b) aspects that suggests external compression (arrows). Note there is no pre-stenotic dilatation of proximal oesophagus.

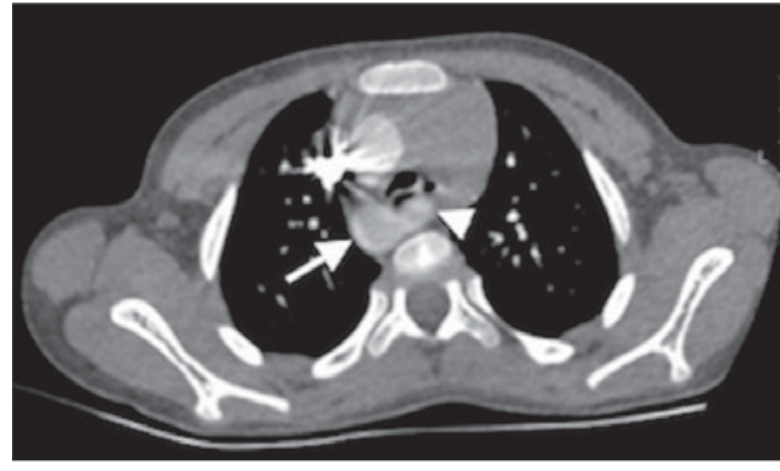

Fig 2: On axial CT Angiograpy image the aortic arch located on right side (arrow) and aberrant left subclavian artery heading towards to left upper extremity after originating from aorta (arrow head) were seen. vertebral column (Figure 3). The findings were consistent with right aortic arch and aberrant left subclavian artery.

The compression of the oesophagus was managed surgically; aberrant left subclavian artery was divided at its origin at the aortic arch and subsequently anostomosed to the left carotid artery. In follow up no complication was encountered, patient could tolerate solid foods well and subsequently started to gain weight.

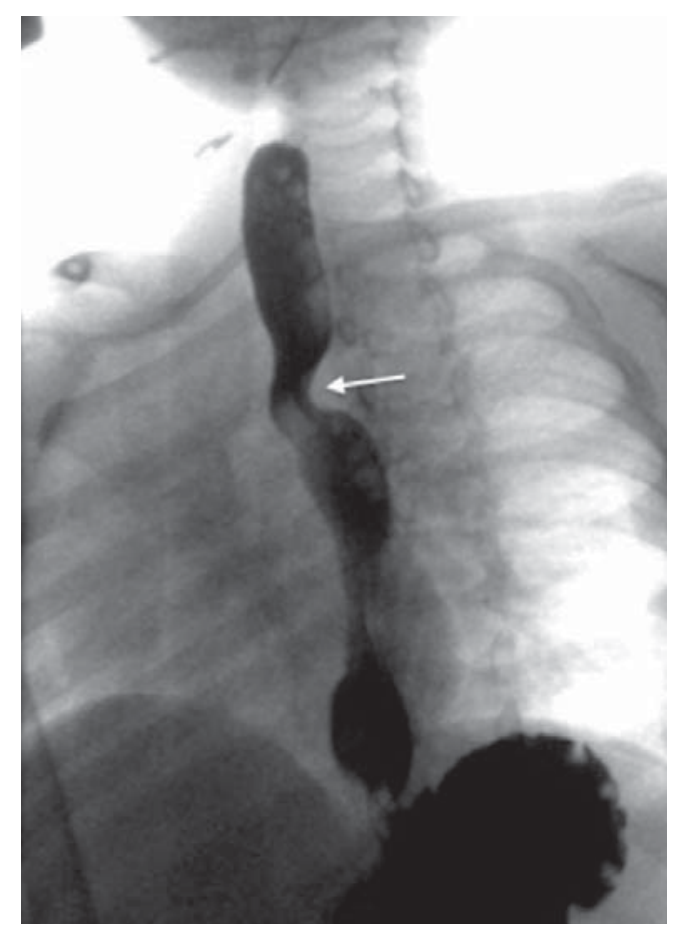




\section{Discussion}

Dysphagia lusoria is a clinical term used to describe the oesophageal dysphagia caused by external compression of oesophagus by vascular structures. It was first described in a woman with dysphagia associated with left aortic arch and aberrant right subclavian artery ${ }^{2}$. Aortic arch developmental anomalies may rarely present with the symptoms of dysphagia in young children ${ }^{3}$. When the oesophageal compression exists, patients often present with dysphagia that starts with the initiation of supplementary food.

There are two aortic arches during fetal life $e^{4}$. The right aortic arch and aberrant left subclavian artery occur due to persistence of right aortic arch and regression of left aortic arch between subclavian and common carotid arteries. Left-sided subclavian artery originates from aortic arch as the last branch and crosses the midline by locating posterior to trachea and oesophagus.

In a patient with the complaint of dysphagia initial imaging work-up to be carried out should be barium esophagography. Indentation of posterior and right side

\section{References}

1. Salanitri J. MR angiography of aberrant left subclavian artery arising from right-sided thoracic aortic arch. $\mathrm{Br} \mathrm{J}$ Radiol 2005;78: 961-66.

2. Bayford D. An Account of a Singular Case of Deglutition. Memoirs Med Soc London 1794;2:271.

3. Donnelly LF, Fleck RJ, Pacharn P, Ziegler MA, Fricke $\mathrm{BL}$, Cotton RT. Aberrant subclavian arteries: Cross sectional imaging findings in infants and children referred for evaluation of extrinsic airway compression. AJR 2002;178:1269-74. of oesophageal wall suggests the presence of right aortic arch and aberrant left subclavian artery. MRA or CTA as the second line imaging modalities may be considered for final diagnosis and pre-operative planning ${ }^{5}$. CTA, with reduced radiation doses in adapted settings for children, may be preferred over MRA, since it enables fast scanning without any necessitation for sedation. In current case, we implemented low dose CTA in order to manifest the type of vascular anomaly.

In the presence of dysphagia the first choice of treatment is surgical decompression procedures if patient's condition allows ${ }^{6}$. Otherwise, the palliative methods as either chewing the solid foods for a long period of time or eating viscous foods are suggested to the patient.

\section{Conclusion}

In paediatric patients, the aortic arch anomalies rarely may lead to the so-called 'dysphagia lusoria'. In order to establish the definite diagnosis CTA or MRA examinations following barium oesophagography should be implemented.
4. Ramos-Duran L, Nance JWJ, Schoepf UJ, Henzler T, Apfaltrer P, Hlavacek AM. Developmental aortic arch anomalies in infants and children assessed with CT angiography Am J Roentgenol 2012;198:466-74.

5. Turkvatan A, Buyukbayraktar FG, Olcer T, Cumhur T. Congenital anomalies of the aortic arch: evaluation with the use of multidetector computed tomography Korean J Radiol 2009;10:176-184.

6. Kogon BE, Forbess JM, Wulkan ML, Kirshbom PM, Kanter KR. Video-assisted thoracoscopic surgery: is it a superior technique for the division of vascular rings in children? Congenit Heart Dis 2007;2(2):130-3. 